(C)2009 IEEE. Personal use of this material is permitted. However, permission to reprint/republish this material for advertising or promotional purposes or for creating new collective works for resale or redistribution to servers or lists, or to reuse any copyrighted component of this work in other works must be obtained from the IEEE. 


\title{
The Power Line Channel Variability
}

\author{
Samir A. Al-Busaidi and Kah-Seng Chung \\ Department of Electrical and Computer Engineering, Curtin University of Technology, \\ Kent Streeet, Bentley, Perth, WA 6102, Australia
}

samir.albusaidi@postgrad.curtin.edu.au and k.chung@curtin.edu.au

\begin{abstract}
This paper describes a flexible way of evaluating the power line (PL) channel frequency response, $H(f)$, and input impedance, in the frequency range of $0-30 \mathrm{MHz}$, by means of a two port network (TPN) methodology. As an example, the method is applied to investigate the power line channel variability under various practical load conditions in a typical modern Australian two bedroom apartment. In order to view the extremities of the channel variability, the channel links have been divided into short and long links. The results show that using short links have the benefits of reduced signal attenuation along with less susceptibility to the variations in the channel conditions.
\end{abstract}

Keywords- Input Impedance, Transfer Function, Indoor Power line communications, PL channel characteristics, MatLab.

\section{INTRODUCTION}

Broadband power line communication (PLC) is gaining momentum as a serious contender for use in home networking. Numerous standards have emerged, such as the HomePlug 1.0 and HomePlug AV [1], while other standards are being forged, like the IEEE 1901 draft standard [2]. All these standards attempt to achieve high throughput on a channel that has been clearly defined as hostile. The increase in achievable data rate has been made possible through a better understanding in the fundamental nature of the power line, PL, channel characteristics, and how to circumvent its hostility.

The PL channel can be viewed, in the most basic of forms, as a number of nodes linked together. A node represents either a general purpose outlet (GPO) or a terminal point. The node links, on the other hand, represents the physically embedded cables. The cables in modern apartments, in turn, consist of a single PVC sheathed live, earth and neutral wire, set side by side in the respective order. The sheathed wires are then held together in place by an outer PVC layer to produce what is known as a flat cable.

To represent such PL channels, many channels models have been proposed. Some resorted to extracting the channel attenuation, delay and weighting parameters from a few measured channel responses [3]. The merit of such an approach is simplicity, but is deficient in accurately representing a broad class of channels. To obtain a broader spectrum of channel responses, others grouped large number of channel measurements into sub-class channel responses that could be further used as representative channels for a wider range of situations [4]. Other authors, in [5], preferred to use a model representing the whole system in terms of cascaded chain scattering matrices. In [6], a two wire indoor PL channel is represented as an ABCD two port network, TPN, which offers flexibility in modeling the response of any channel topology once the complete electrical power wiring circuit is specified.

This paper presents a computer simulation study on the power line channel variability of a two-bedroom apartment using a Matlab computer program, written based on the use of the TPN approach. The variations in amplitude response as well as input impedance of the power line channel in the presence of different combinations of typical home appliances are examined. Section 2 describes the typical channel network topology of a modern two-bedroom apartment. This is followed by a discussion in Section 3 on the use of the ABCD two-port network to model of a given PL channel. Section 4 presents the simulation results. Some conclusions are drawn in Section 5 .

\section{ELECTRICAL WIRING IN A TWO-BEDROOM APARTMENT}

An example of a typical modern Australian two bedroom apartment electrical power circuits wiring diagram is shown in Figure 1. With modern Australian electrical practices, the mains power supplies enter a household premise at the service panel (SP) node, node 6. Stemming from the SP node are individual circuits on which all electrical appliances are connected to. In the example, there are three general purpose circuits, each having numerous GPOs. These circuits are indicated as circuits 1,2 and 3, with the GPOs shown as nodes on each of the individual circuits. In addition, there are other independent circuits that connect to the SP. Each of these circuits supports a single high current electrical appliance, such as a wall oven, water heater or hot plate. These circuits are shown in the Figure 1 as circuits 4, 5 and 6, respectively.

Cables connected to a GPO are dropped from the false ceiling, down the face of the wall to the level of the GPO before being looped again up into the ceiling. Such is the general modern practice found in Australian high rise construction sites. With an average ceiling height of $2.5 \mathrm{~m}$, it can be assumed that any two adjacent GPOs should have a minimum separation of approximately $5 \mathrm{~m}$. 


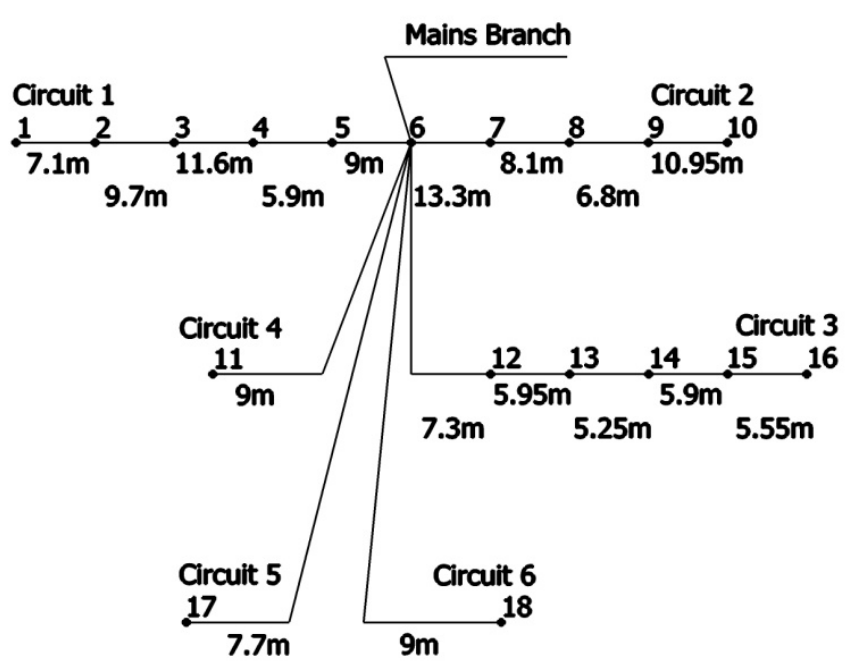

Figure 1. Power line wiring in a typical two bedroom apartment.

Appliances are generally plugged into the GPO of a circuit through a connecting cable of a certain finite length. In order to distinguish the cables that are embedded in the building to those that are extended from the appliances, the former will be referred from here on as cables whilst the latter as cords.

In a real scenario, a PL channel may be made up of different types of cables and cords, each having different lengths. Although the developed Matlab simulator can take into consideration all these different scenarios, two assumptions are made for all that follows. First, the maximum length of a connecting cord is set to $4 \mathrm{~m}$. Secondly, all cords and cables that are used in the simulation are of the same type.

In an attempt to produce results that are representative of a real indoor PL channel, a given electrical appliance is characterized in terms of its equivalent impedance made up from a series resistor and inductor [9]. Two types of appliances have been identified, those that are purely resistive and those that are complex. An example of a complex load appliance is a motor, which can be represented as a resistor in series with an inductor.

From another perspective, a PL channel is formed by connecting a transmitter and a receiver to two different nodes or GPOs, which may be either on the same or a different circuit. Also, it is likely that other electrical appliances may be connected to some of the GPOs located in between the transmitter and receiver. Such a PL channel can be viewed in terms of a tree structured network. In this case, each individual branch of the network may be represented by an equivalent impedance looking into that particular branch. The influence of any branch can be then taken into account in the computation of the channel response as described in the next section.

\section{TPN THEOREM}

The output voltage, $V_{2}$, and current, $I_{2}$, of a two port network are related to its input voltage, $V_{l}$, and current, $I_{l}$, by an ABCD matrix, such that,

$$
\left[\begin{array}{l}
V_{2} \\
I_{2}
\end{array}\right]=\left[\begin{array}{ll}
A & B \\
C & D
\end{array}\right]\left[\begin{array}{l}
V_{1} \\
I_{1}
\end{array}\right]=\Psi\left[\begin{array}{l}
V_{1} \\
I_{1}
\end{array}\right]
$$

Now, consider a two wire transmission line that connects two nodes, nodes $i$ and $j$, as shown in Figure 2a. This transmission line can be expressed in terms of an ABCD matrix, such that

$$
\begin{aligned}
& \psi_{t_{t}}=\left[\begin{array}{ll}
A_{t} & E_{t} \\
C_{t j} & D_{t}
\end{array}\right]
\end{aligned}
$$

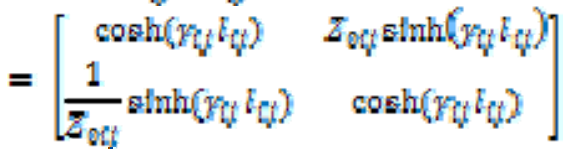

where $Z_{0 i j}$ and $\gamma_{i j}$ are the characteristic impedance and propagation constant of the transmission line of length $l_{i j}$, respectively.

If the transmission line is terminated with a load, then the equivalent impedance looking into node $i$ is given by

$$
z_{z q}^{i j}=\frac{A_{l j} z_{l j}+B_{l j}}{C_{l j} z_{l j}+D_{l j}}
$$

Such an equivalent representation of a transmission line section with a termination load is shown in Figure $2 b$.

From Figure 1, a simple PL channel may be formed by connecting one or more transmission line sections in series. As such, the overall ABCD matrix, ,which represents

$N$ transmission line sections in cascade is

$$
\Psi_{\text {Comi, }, 8}=\Psi_{1,2} \Psi_{2,8} \ldots \Psi_{W-1, W}
$$

where is the $\mathrm{ABCD}$ matrix for the transmission line connecting nodes $(\mathrm{N}-1)$, and $\mathrm{N}$.

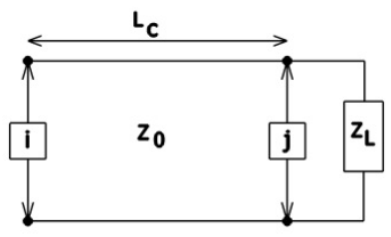

(a)

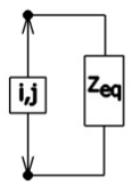

(b)

Figure 2. Equivalent two wire line representations, (a) Physical connection (b) Equivalent representation.

Next, consider the case when an appliance is connected via a cord of length $L_{c}$ to a node, say, node $i$, which lies within a PL channel. This cord and appliance combination can be modeled as in Figure 2. The resultant equivalent impedance 
is now considered to be connected in parallel to the node $i$. As a result, the $\mathrm{ABCD}$ matrix that represents this parallel impedance is given by

$$
\begin{array}{r}
\psi_{l}^{r}=\left[\begin{array}{cc}
1 & 0 \\
1 / z_{e q}^{l} & 1
\end{array}\right] \\
=\left[\begin{array}{ll}
1 & 0 \\
\frac{C_{q d} z_{a}+D_{q d}}{A_{g d} z_{a}+B_{e d}} & 1
\end{array}\right]
\end{array}
$$

where $Z_{a}$ is the impedance of the appliance, and the ABCD matrix of the cord is denoted with the subscript "cd".

The above same approach is also applicable to situations where there are multiple parallel branches connected to a given node, such as the case with several circuits stemming out from the service node SP. Let the equivalent impedance looking into a parallel branch, say branch $m$, be Then, the resultant $\mathrm{ABCD}$ matrix by combing all the $\mathrm{M}$ parallel branches, looking from the node where these branches are connected to, is given by

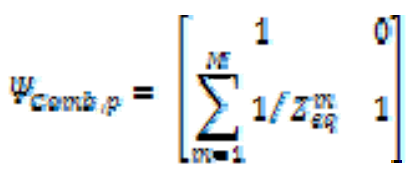

Finally, a PL channel can be modeled as a cascade of transmission line sections, each represented by its ABCD matrix. As a result, the overall $\mathrm{ABCD}$ matrix representing the PL channel can be expressed as

$$
\Psi_{T a t}=\Psi_{A} \Psi_{Q} \Psi_{S} \ldots
$$

Incorporating the source and load impedance, $\mathrm{Z}_{\mathrm{S}}$ and $\mathrm{Z}_{\mathrm{L}}$, respectively, the channel transfer function (TF), $H(f)$, and input impedance, $Z_{\text {in }}$, are computed according to

$$
\begin{aligned}
& R(f)=\frac{Z_{L}}{A \Sigma_{L}+B+C \Sigma_{L} Z_{S}+D Z_{I}} \\
& z_{n}(f)=\frac{A Z_{L}+B}{C Z_{L}+D}
\end{aligned}
$$

\section{COMPUTER SIMULATION}

Based on the two-port approach described in Section III, a PL channel evaluation software has been written in Matlab to compute the channel transfer function and input impedance. The required input parameters for the channel evaluator are the node numbers for the transmitter and receiver, the transmit source impedance and receive load impedance, the interconnecting node numbers, the location of the SP node, the corresponding cable types and lengths, and the appliance impedances and associated node numbers. The channel evaluation begins by determining the direct path between the Tx and Rx and all the branches that stem from the direct path. It then proceeds to compute, in a systematic fashion, the equivalent two-port ABCD matrix of each link section from the Tx towards the Rx for every specified frequency up to 30 $\mathrm{MHz}$. Once the overall ABCD matrix for the PL channel is determined, equations (8) and (9) are then used to compute its $H(f)$ and $Z_{\text {in }}(f)$, respectively.

Referring to the wiring connections of Figure 1, the power distribution is such that circuit 1 feeds the first bedroom and bathroom areas, circuit 2 supplies the second bedroom, bathroom and living areas, while circuit 3 caters for the kitchen area. Although all the three circuits are equipped with GPO points, some of these GPOs are not likely to be connected to a transmitter and receiver because of their unsuitable or inconvenient locations. For example, having transmitter or receiver connected to the GPOs in bathrooms. On the other hand, some GPOs may likely be permanently connected to larger appliances, such as washing machine, fridge and dryer. As such, they are also not available for used by the transmitter or receiver. Table I shows the more likely GPOs that could form a $T x / R x$ pair. Each $T x / R x$ pair is assigned a link number together with the node numbers of the transmitter and receiver. As a PL channel is considered to be reciprocal [8], this suggests that the locations of the transmitter and receiver can be interchanged.

TABLE I.

Tx/Rx LINKS and CORRESPONDING NODE PLACEMENTS

\begin{tabular}{|c|c|c|}
\hline Link Number & Tx Node & Rx Node \\
\hline 1 & 1 & 2 \\
\hline 2 & 1 & 4 \\
\hline 3 & 1 & 5 \\
\hline 4 & 1 & $\mathbf{8}$ \\
\hline 5 & 1 & 10 \\
\hline 6 & 2 & 4 \\
\hline 7 & 2 & $\mathbf{8}$ \\
\hline 8 & 2 & 10 \\
\hline 9 & 2 & 5 \\
\hline 10 & 4 & $\mathbf{8}$ \\
\hline 11 & 4 & 10 \\
\hline 12 & 4 & \\
\hline
\end{tabular}

\section{RESULTS}

The aim of this investigation is to determine the variability of a PL channel, in terms of its amplitude response $H(f)$, and input impedance $Z_{\text {in }}(f)$, when various electrical appliances and circuits are connected. This is demonstrated by considering a short (link 1) and a long (link 8) links. In the case of the short link, the transmitter and receiver share the same circuit whilst for the long link they are located on two different circuits.

To simplify simulations described in this paper, it is assumed that cables and cords are going to have the same and , namely, Ohms and = 4.7224 ns. In practice, the power cables and connecting cords are generally of different constructions having different values of and Also, it is assumed that the transmitter output impedance, and receiver input impedance are matched to the $140 \mathrm{Ohm}$ cable.

First, the response of a PL channel under the influence of individual circuits stemming from the SP node is investigated. The results are shown in Figure 3 for a short PL link, and 
Figure 4 for a long PL channel. These results include the channel response of a straightforward PL link with no other branches at the SP node. The channel response is then computed by connecting an additional circuit, either 3 , or 4 , or 5 at the SP node. Finally, the response of the PL channel with all the branch circuits connected at the SP node is plotted as the solid-line curve in Figures 3 and 4. Unless otherwise stated, all GPOs are left unconnected.

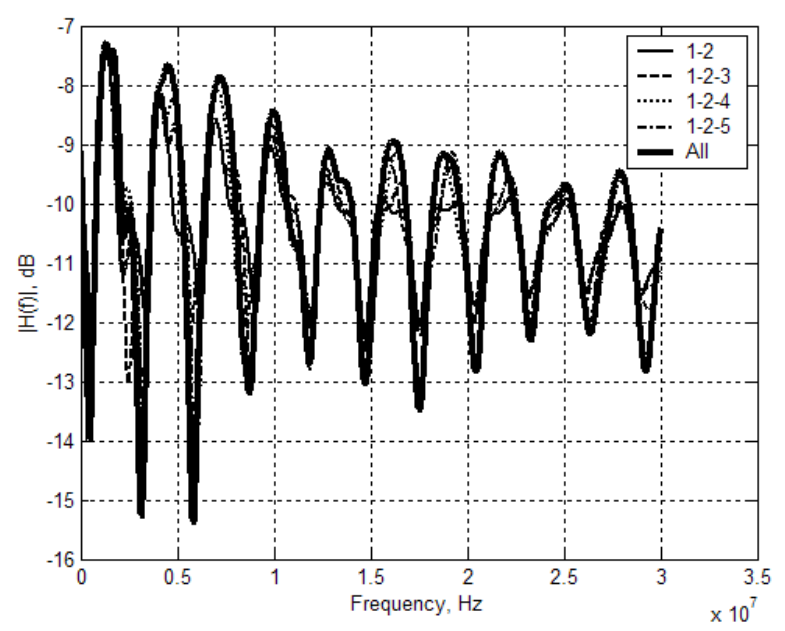

Figure 3. Influence of branch circuits at SP node on the frequency response of the short Tx/Rx link (Link 1).

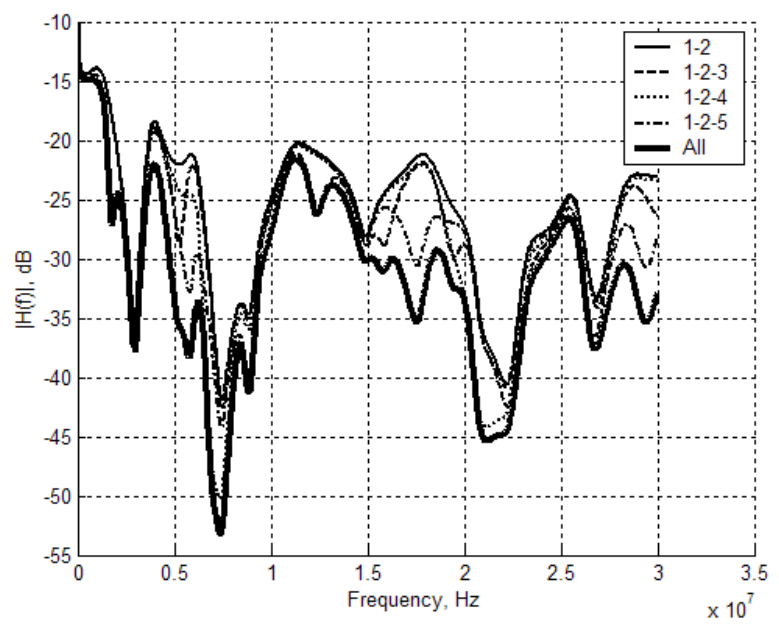

Figure 4. Influence of branch circuits at SP node on the frequency response of the long Tx/Rx link (Link 8).

Figures 3 and 4 show that both the short and long PL links are highly frequency selective, i.e., with deep notches occurring in the channel responses. As expected, the shorter link (Link 1) suffers from lower attenuation than the longer link (Link 8). For the short link, the SP node is located outside and some distance away from it. As a result, its channel response is almost not affected by having an additional power circuit connected to the SP node. On the other hand, Link 8 incorporates the SP node within its path. As such, the addition of a branch circuit at the SP node will affect the channel response of Link 8 significantly.
Next, the effect of connecting an appliance to a GPO via a connecting cord on the frequency response of a PL channel is investigated. The cord length used ranges from 0 to 4 meters. A list of appliances considered is tabulated in Table II together with their respective impedances [9]. The channel response is computed by connecting the same appliance to all the GPOs of circuits 1, 2 and 3. This has been carried out for the first three appliances listed in Table II. The water heater, wall oven, and hot plate are permanently connected to circuit 4, 5 and 6 , respectively. From these channel responses, the minimum and maximum values at a given frequency are collected and plotted in Figure 5 for the short PL channel (Link 1), and Figure 6 for the long PL channel (Link 8). For comparison, a selected number of curves depicting an appliance connected to a certain nodes, while all the remaining GPOs are left unconnected is also shown in Figures 5 and 6.

TABLE II.

VARIOUS ELECTRICAL APPLIANCES EQUIVALENT IMPEDANCE

\begin{tabular}{|c|c|}
\hline Appliance & $\begin{array}{c}\text { Equivalent impedance } \\
\text { (Ohms @ 60 Hz) }\end{array}$ \\
\hline Radio clock & $\mathbf{1 1 5 2 0}$ \\
\hline TV & $\mathbf{1 9 2}$ \\
\hline Heater & $\mathbf{2 3 . 5}$ \\
\hline Hot plate & $\mathbf{1 9 2}$ \\
\hline Wall Oven & $\mathbf{6 . 8 5}+\mathbf{j} 19.7$ \\
\hline Water Heater & $\mathbf{1 4 . 4}$ \\
\hline
\end{tabular}

There is a notable variation in the TF for both links. The minimum TF clearly retains the main notches of the original link $\mathrm{TF}$, but at a significantly attenuated level. The reasoning behind this, from one side, lies in that the appliance cord length is too short to imprint within the $30 \mathrm{MHz}$ bandwidth any new notches. From the other side, the large mismatches of the appliances and the rest of the PL network can introduce significant destructive multipath interference. However, although there can be significant signal attenuation, this may be viewed as an overly pessimistic scenario of a single appliance.

For the case of the short link (Link 1), it is clear that the minimum values are attained when the heater is connected to node 2. Connecting an appliance to any other nodes, such as nodes 3 and 4, show that the losses in the TF are less by approximately $10 \mathrm{~dB}$. Node 2 , for this link, is the only node that resides between the transmitter/receiver, $\mathrm{Tx} / \mathrm{Rx}$, pair.

In the case of the long link (Link 8), there are a number of nodes that are responsible for the large $\mathrm{TF}$ variability. In particular, connecting an appliance on nodes 4 and 5 is going to lower the overall TF. On the other hand, an appliance connected to node 12 will not vary the TF to the lowest level. This suggests that the resulting minimum $\mathrm{TF}$ variation is largely due to an appliance connected in between the $\mathrm{Tx} / \mathrm{Rx}$ pair, nodes 4 and 5 for the long link and node 2 in the short link (Link 1). 


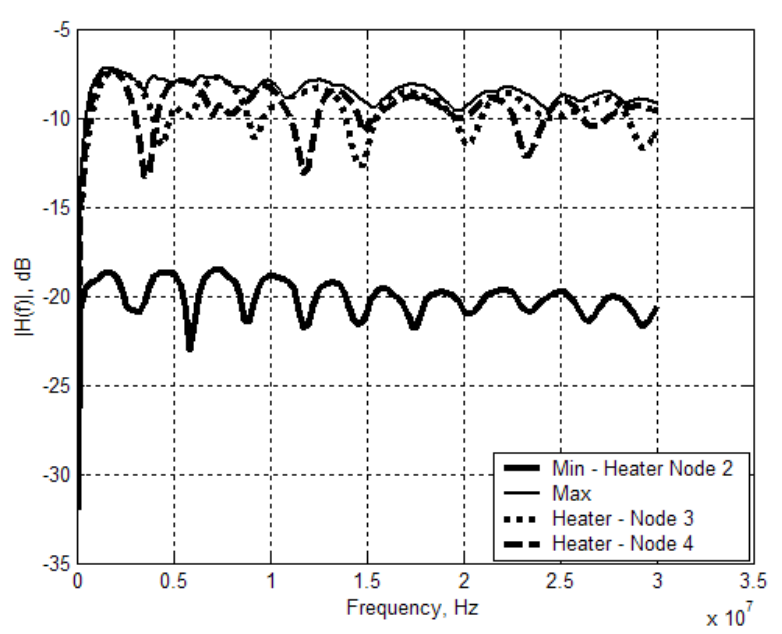

Figure 5. Maximum and minimum values of the channel response of the short link in the presence of single appliances connected to a cord of different lengths.

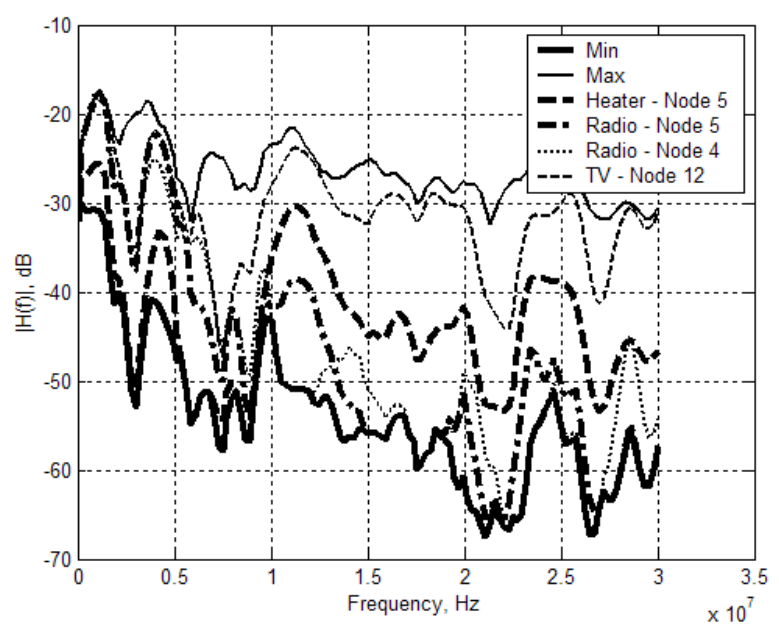

Figure 6. Maximum and minimum values of the channel response of the long link in the presence of single appliances connected to a cord of different lengths.

To encompass the true nature of a complete PL channel, 200 different channel configurations for each of the links given in Table 1 were simulated. In these simulations certain GPOs were reserved for specialized appliances, such as washing machines, dishwashers and kitchen appliances. For the rest of the GPOs a variety of other commonly found home appliances were used. For each GPO appliance the cords were varied randomly according to a uniform distribution, in the range of $1 \mathrm{~m}$ to $4 \mathrm{~m}$.

Figure 7 represents the maximum, minimum and root mean square, RMS, TF for the short and long links. The short link (Link 1) shows significantly less variation in comparison to the long link (Link 8). The effect of having just a single node that would really significantly change the $\mathrm{TF}$, node 2 , is the primary reason for the short link maintaining an approximate $20 \mathrm{~dB}$ variation over the PL bandwidth. In contrast, the long link shows a significantly greater variability that can peak to
$45 \mathrm{~dB}$. This again is attributed to the number of GPO nodes that are connected the direct $\mathrm{Tx} / \mathrm{Rx}$.

The resulting RMS, channel response of all the links given in Table 1 is depicted in Figure 8. The curves in the Figure can be sub-divided into two broad groups. Consider all curves that are above $-30 \mathrm{~dB}$ at $\mathrm{DC}$ in one group and the rest to be in the other group. The resulting subdivision is:

Growp $1=\operatorname{Links}\{1,10,6,2,7,3\}$
Growp $2=\operatorname{Lthks}\{5,9,4,8,12,11\}$

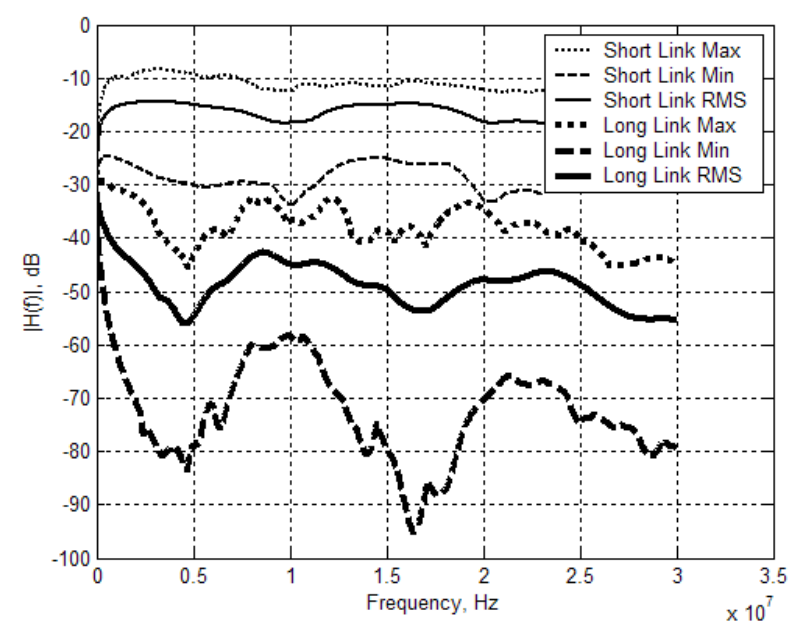

Figure 7. Link TF variations for multi-appliance configurations.

All the links in group 1 reveal that the Tx/Rx pair is placed on the same circuit. Group 2, on the other hand, represents links where the $\mathrm{Tx} / \mathrm{Rx}$ pair is placed on different circuits. The minimum RMS obtained from group 1 is marginally less attenuated than the maximum RMS TF of group 2. It can thus be deduced that if a Tx/Rx pair are located on the same circuit, the attenuation will generally be smaller than if the pair were on different circuits.

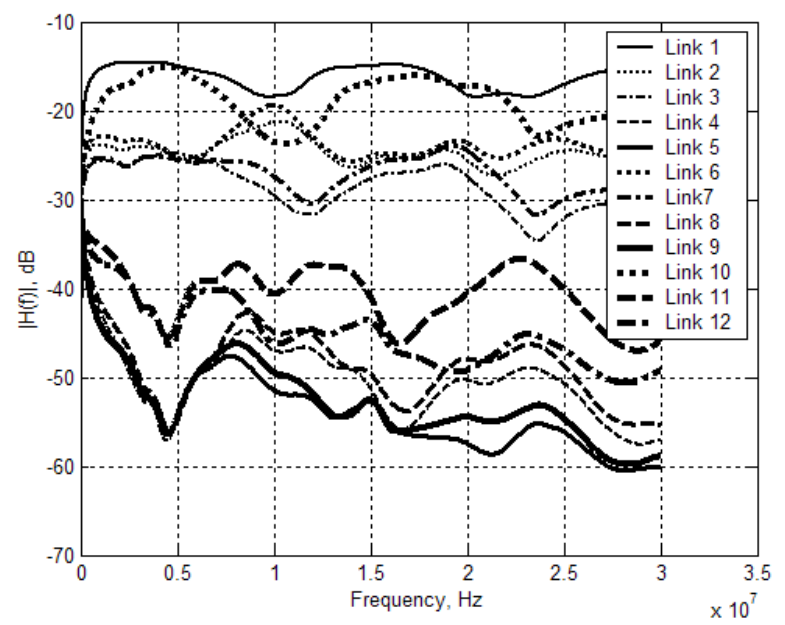

Figure 8. $|\mathrm{H}(\mathrm{f})| \mathrm{RMS}$ values for the different $\mathrm{Tx} / \mathrm{Rx}$ links.

Upon closer inspection of the curves in group 2 reveal a persistent notch at approximately $4.5 \mathrm{Mz}$. This is then followed by another notch at $16 \mathrm{MHz}$. The notches are attributed to the average appliance cord length of 2.5 meters, 
compounded by the number of appliances that resides between the $\mathrm{Tx} / \mathrm{Rx}$ pair. These appliances jointly introduce destructive interference at the specified frequencies.

In Figure 9 the RMS value of the input impedance for all the $\mathrm{Tx} / \mathrm{Rx}$ pairs is shown. It is clear that the input impedance for each link varies differently. However, the plot of the mean shows a smooth curve that may be implementable for matching purposes. The mean curve shows good consistency to the measured results in [10].

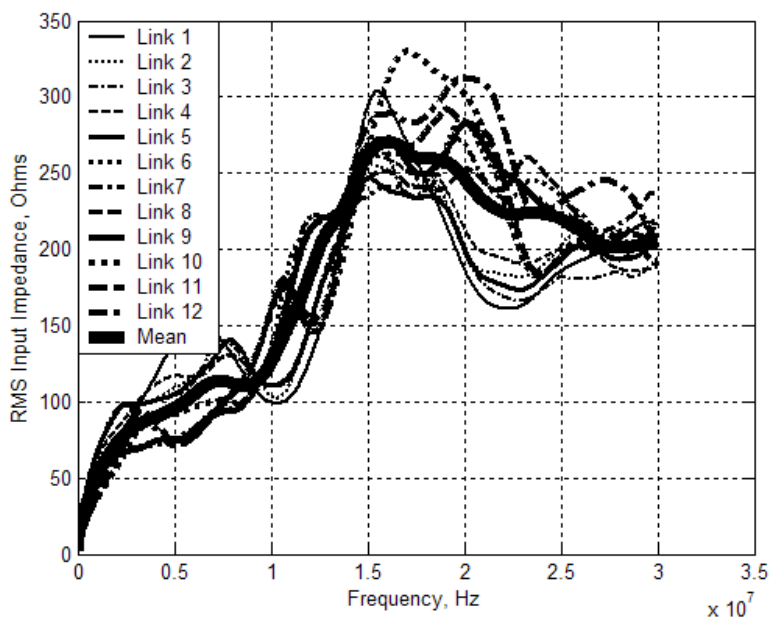

Figure 9. RMS $|\mathrm{Zin}|$ value for the different $\mathrm{Tx} / \mathrm{Rx}$ links.

\section{CONCLUSION}

In this paper, the variability of the channel response of a PL in a typical modern two bedroom apartment has been investigated, using a custom written evaluation software in Matlab. The evaluation, over a frequency range of up to 30 $\mathrm{MHz}$, includes the study on the influence of various factors, such as the length of the links, the presence of multiple branch circuits and the presence of various appliances connected to GPOs with different cord lengths.

It is observed that a PL channel is highly sensitive to where the transmitter and receiver are located. The frequency response of a PL channel tends to suffer from losses as well as deep and wide notches when various appliances are connected to it. The presence of such notches indicates that a PL channel is generally frequency selective. However, the frequency selective effect is reduced by placing the transmitter and receiver at the end GPOs of a power circuit.

For the wiring connections of the apartment, as shown in Figure 1, there different branch connections are identified. These are (i) a branch that connects to a node located in between the transmitter and receiver, (ii) a branch that connects directly to either the transmit or receive node, and (iii) a branch that joins another branch which is connected to a node along the path of the transmitter and receiver. Each of these three branch connections may cause a different degree of variability to a PL channel. The first type of branch connections tends to cause the most severe channel variability. The second branch type is the result of the transmitter or receiver not being connected to an end node. Such branch link gives rise to a lesser degree of channel variability compared with the first type of branch connections. Finally, the third branch type does not show much influence on the channel variability.

The frequencies where the various notches occurred in the frequency response are dependent on the branch circuit length. For PL channels with long branching circuits, it is observed that many shallow notches are present in their respective frequency responses.

Finally, an rms impedance curve has been obtained based on the input impedances of many different transmit-receive link configurations. It is observed that the input rms impedance curve tends to increase with frequency up to 15 $\mathrm{MHz}$, after which it falls off gradually until $30 \mathrm{MHz}$. This observation may provide some useful hints in how to match a PL channel.

\section{REFERENCES}

[1] http://www.intellon.com/technology/standards.php

[2] http://grouper.ieee.org/groups/1901/

[3] Dostert, K. and Zimmermann, M., "A Multipath Model for the Powerline Channel", IEEE Trans on Communications, Vol. 50, No. 4, April 2002.

[4] Tlich, M. et al, "A Broadband Powerline Channel Generator", ISPLC'07, 26-28 March 2007, Pisa, Italy.

[5] Meng, H. et al, "Modeling of Transfer Characteristics for the Broadband Power Line Communication Channel", Trans on Power Delivery, Vol. 19, No. 3, July 2004.

[6] Li. H., et al, "The Indoor Power Line Channel Model Based on Twoport Network Theory", ICSP2008 Proceedings, 10-11 May, 2008, Leipzig, Germany.

[7] Banwell, T. and Galli, S., "A Deterministic Frequency-Domain Model for the Indoor Power Line Transfer Function", IEEE J. on Selected Areas in Communications, Vol. 24, No. 7, July 2006.

[8] Banwell, T. and Galli, S., "A Novel Approach to the Modeling of the Indoor Power Line Channel Part II: Transfer Function and its Properties", IEEE Trans on Power Delivery, Vol. 20, No. 3, July 2005.

[9] www.askthebuilder.com/B178_Common_Wattage_of_Household_Appl iances.shtml

[10] Tang, L. T., et al, "Characterization and Modeling of In-Building Power Lines for High-Speed Data Transmission", IEEE Trans on Power Delivery, Vol. 18, No.1, Jan 2003. 